\title{
Surface Condition and Conductance of Graphene in Redox Process
}

\author{
Received 5 October, 2021; revised 9 October, 2021; accepted 15 October, 2021
}

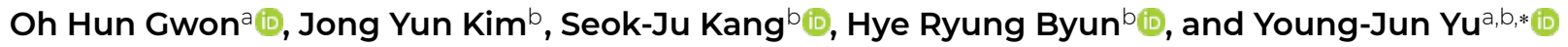 \\ a Department of Physics, Chungnam National University, Daejeon 34134, Republic of Korea \\ bInstitute of Quantum Systems, Chungnam National University, Daejeon 34134, Republic of Korea
}

*Corresponding author E-mail: yjyu@cnu.ac.kr

\begin{abstract}
In this study, we investigated the redox process occurring on graphene with an $\mathrm{H}_{2} \mathrm{SO}_{4}$ electrolyte gating. The redox process occurring on graphene was studied by observing the variation in the conductance of graphene. By performing electric force microscopy measurements, we also observed the surface condition of graphene leading oxidation and its role as a seed for adsorbing undesired residues, which results in conductance suppression.
\end{abstract}

Keywords: Graphene, Graphene oxide, Electrolyte gate, Oxidation, Reduction, Redox

\section{Introduction}

Selective oxidation on the surface of graphene using electrochemical techniques has been suggested for various applications, such as chemical gas sensing and biosensing due to its high sensitivity for sensing target molecules as well as the high surface-to-volume ratio of twodimensional carbon networks [1-14]. Oxidation on graphene with an electrolyte gating is one of the well-known methods for functionalization on graphene. Although various techniques are available for oxidation on graphene, electrochemical oxidation has been favored as it enables a reversible process between graphene and graphene oxide [15-20]. Since this oxidation and reduction (i.e., redox) process on graphene allows for tuning of the graphene conductance over a wide range, it has been employed to develop graphene electrodes with the required conductance [15-23]. Meanwhile, continuous conductance degradation of graphene during the redox process has been reported, and this conductance suppression should be investigated with oxidized condition $[3,19-24]$. In this study, we performed the redox process on graphene using an electrolyte gating. Using a sulfuric acid $\left(\mathrm{H}_{2} \mathrm{SO}_{4}\right)$ electrolyte, graphene was oxidized, and the resulting graphene oxide was reduced while observing the conductance variation of the graphene. The graphene surface was observed using electric force microscopy (EFM) measurements. These investigations allowed us to confirm the oxide seed formation on graphene and its role in extending the adsorption of undesired residues on graphene, which leads to a substantial conductance reduction.

\section{Experimental details}

Graphene was fabricated on a 280 -nm-thick $\mathrm{SiO}_{2}$ substrate via mechanical exfoliation and was contacted with titanium (Ti) electrodes via a normal e-beam lithography process [Fig. 1(a)] [19-25]. Ti forms a thin oxide layer $\left(\mathrm{TiO}_{2}\right)$ naturally in air, which protects the Ti electrodes from the $\mathrm{H}_{2} \mathrm{SO}_{4}$ electrolyte. To use one of the electrodes as electrolyte gate metal, we disconnected the metal electrode from graphene through an oxygen plasma etching process using a poly methyl metha- (a)
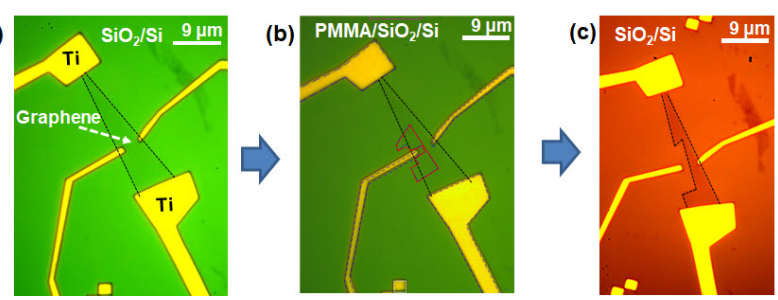

Figure 1. (a) Optical image of graphene contacted with Ti electrodes on $\mathrm{SiO}_{2} / \mathrm{S}$ substrate. (b) Optical image of PMMA mask on graphene-Ti electrodes on $\mathrm{SiO}_{2} / \mathrm{Si}$ substrate. The red dashed area denotes an opened PMMA mask window. (c) Optical image of graphene contacted with Ti electrodes on $\mathrm{SiO}_{2} / \mathrm{Si}$ substrate after oxygen plasma etching and PMMA mask removal.

crylate (PMMA) etch mask, as shown in Figs. 1(b) and 1(c).

The conductance of graphene was determined using two-terminal measurement with standard lock-in techniques under an $\mathrm{H}_{2} \mathrm{SO}_{4}(1$ M) electrolyte-gated voltage $\left(\mathrm{V}_{\mathrm{EG}}\right)$ through the Ti electrode, which was located beside the graphene [Fig. 2(a)]. We employed $\mathrm{H}_{2} \mathrm{SO}_{4}(1$ $\mathrm{M})$ as the electrolyte for characterizing the redox process on graphene because this type of electrolyte gating guarantees conductance tuning as well as a reproducible redox process for graphene, as observed in our previous studies $[19,20,22]$.

\section{Results and discussion}

In Fig. 2(b), we observed a conductance of $\sim 31.5 \mu \mathrm{S}$ for $\mathrm{V}_{\mathrm{EG}}=0$ $\mathrm{V}$ (marked by red dashed arrows) and a conductance range of 30-32 $\mu \mathrm{S}$ when $\mathrm{V}_{\mathrm{EG}}$ varied from 0.6 to $-0.4 \mathrm{~V}$. Since the capacitance of the electrolyte gate (several hundreds $\mathrm{nF} / \mathrm{cm}^{2}$ ) is around ten times that of $280 \mathrm{~nm}$ thick $\mathrm{SiO}_{2}$ (several tens of $\mathrm{nF} / \mathrm{cm}^{2}$ ) [19-22], we could tune the conductance of graphene for $\mathrm{V}_{\mathrm{EG}}$ values less than $1 \mathrm{~V}$.

Figure 3 illustrates the electrolyte-gated conductance variations and oxidation of graphene. After dropping sulfuric acid liquid solution $\left(\mathrm{H}_{2} \mathrm{SO}_{4}, 1 \mathrm{M}\right)$ onto the graphene and the gated electrode area, we measured the conductance of the graphene by tuning $\mathrm{V}_{\mathrm{EG}}$ as shown in Fig. 

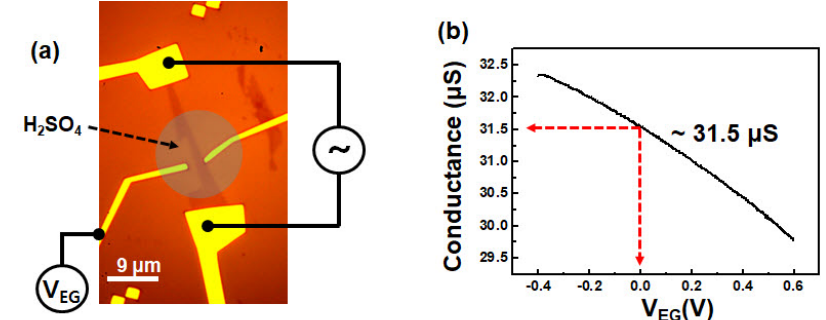

Figure 2. (a) Schematic image of $\mathrm{H}_{2} \mathrm{SO}_{4}$ electrolyte gate through graphene-Ti electrodes on $\mathrm{SiO}_{2} / \mathrm{Si}$ substrate. (b) Conductance of graphene as a function of electrolyte gate voltage $V_{E G}$. The red dashed arrows indicate the conductance of graphene $(\sim 31.5 \mu \mathrm{S})$ for $\mathrm{V}_{\mathrm{EG}}=0 \mathrm{~V}$.

3(a). We observed that the conductance increased as $\mathrm{V}_{\mathrm{EG}}$ was gradually changed from 0 to $-1.90 \mathrm{~V}$; thereafter, the conductance rapidly decreased to $0 \mu \mathrm{S}$ for $\mathrm{V}_{\mathrm{EG}}=-1.95 \mathrm{~V}$, as shown in Fig. 3(b). Thus, the $p$-doping enhancement of graphene led to a conductance improvement when $\mathrm{V}_{\mathrm{EG}}$ was between 0 and $-1.90 \mathrm{~V}$, and subsequently, the oxidization on the graphene surface resulted in a conductance suppression when $\mathrm{V}_{\mathrm{EG}}$ was $-1.95 \mathrm{~V}$. This indicates that $\mathrm{V}_{\mathrm{EG}}=-1.95 \mathrm{~V}$ is the critical potential for initiating oxidation on the graphene surface with $\mathrm{H}_{2} \mathrm{SO}_{4}$ electrolyte. To determine the surface potential condition of graphene, we studied the EFM image of graphene after the electrolyte gating process (1); the results are shown in Fig. 3(b). The spatial electrical surface potential distribution of graphene was observed by applying a drain-source bias voltage of $\mathrm{V}_{\mathrm{DS}}=-1 \mathrm{~V}$ along different directions, as shown in Figs. 3(c) and 3(d). To determine the surface potential, we performed EFM measurement using commercial atomic force microscopy (AFM; XE-100, Park Systems Co.) [20,25-27]. During the measurement, an $\mathrm{AC}$ voltage of $0.5 \mathrm{~V}$ with an amplitude of 17
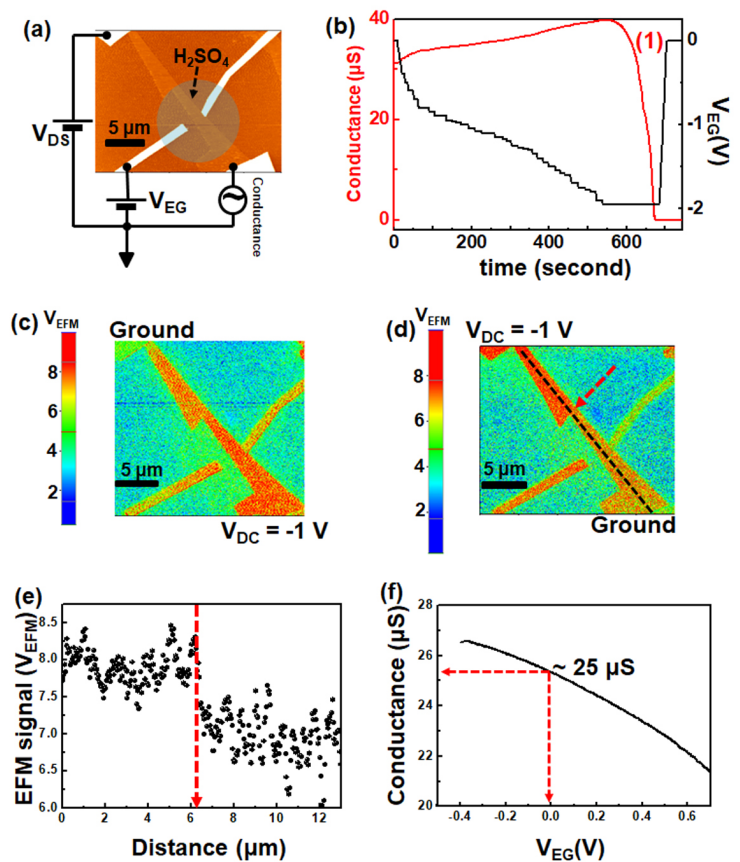

Figure 3. (a) Schematic of conductance tuning and oxidation of graphene with $\mathrm{H}_{2} \mathrm{SO}_{4}$ electrolyte. (b) Conductance variation of graphene as a function of time while sweeping $\mathrm{V}_{\mathrm{EG}}$ from 0 to $-1.95 \mathrm{~V}$. EFM $\left(\mathrm{V}_{\mathrm{EFM}}\right)$ images of graphene after oxidation process (1) in (b), upon applying bias voltage $\mathrm{V}_{\mathrm{DS}}=-1 \mathrm{~V}$ on the (c) top and (d) bottom electrodes. (e) EFM image profile following along the black dashed line in (d). The red dashed arrow denotes the same as position as in (d). (f) Conductance of graphene as a function of electrolyte gate voltage $V_{E G}$. The red dashed arrows indicate the conductance of graphene $(-25 \mu \mathrm{S})$ for $\mathrm{V}_{\mathrm{EG}}=0 \mathrm{~V}$.
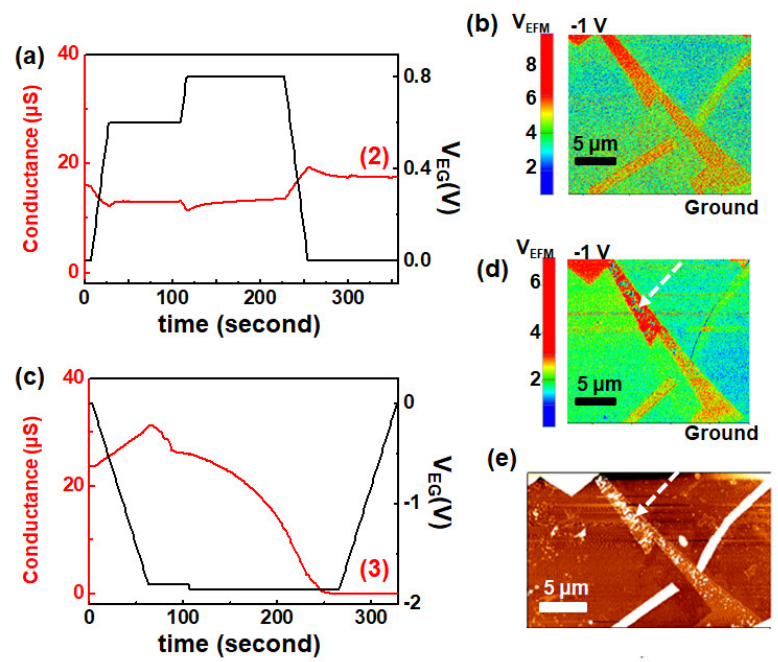

(d)

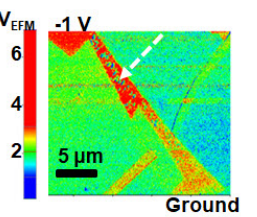

(e)

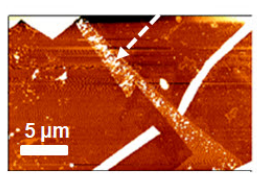

Figure 4. (a) Conductance variation of graphene as a function of time while sweeping $V_{E G}$ from 0 to $0.8 \mathrm{~V}$. (b) EFM $\left(V_{\text {EFM }}\right)$ image of graphene after oxidation process (2) in (a), upon applying $V_{D S}=-1 \mathrm{~V}$. (c) Conductance variation of graphene as a function of time while sweeping $V_{E G}$ from 0 to $-1.80 \mathrm{~V}$. (d) EFM $\left(V_{\mathrm{EFM}}\right)$ image of graphene after oxidation process (3) in (c), upon applying $\mathrm{V}_{\mathrm{DS}}=-1 \mathrm{~V}$. The white dashed arrow denotes undesired residues on graphene. (e) Topography image of graphene after oxidation process (3) in (c). The white dashed arrow denotes undesired residues on graphene.

$\mathrm{kHz}$ was applied to the metal-coated cantilever probe, and the topography image was measured in the noncontact mode with a $170 \mathrm{kHz}$ oscillating cantilever. Furthermore, to initiate an electrostatic interaction between the metal tip and graphene surface, we applied a DC voltage of $1.0 \mathrm{~V}$ to the metal cantilever. As the EFM signal $\left(\mathrm{V}_{\mathrm{EFM}}\right)$ in this study reflects the difference between the electric potential of the metal tip and that of the graphene (which has a background potential), the $V_{\text {EFM }}$ values could be used to identify only the electrostatic variation on the graphene surface during the redox process. As a result, the EFM images of graphene [Figs. 3(c) and 3(d)] depict the electrostatic potential gradient from $\mathrm{V}_{\mathrm{DC}}=-1 \mathrm{~V}$ to the ground. In Fig. 3(e), the $\mathrm{V}_{\text {EFM }}$ profile follows the length of the graphene [marked with the black dashed line in Fig. 3(d)] and clearly shows the electrostatic potential drop at the boundary [marked with the red dashed arrow in Fig. 3(e)], which has the smallest width [marked with the red dashed arrow in Fig. 3(d)]. This shows that applying $\mathrm{V}_{\mathrm{DC}}$ along different directions in graphene leads to suitable current flow, with the bottleneck area causing a potential drop. Although this result suggests that the oxidation is not strong on the graphene surface during the oxidation process (1) depicted in Fig. 3(b), strong disordered areas still exist on the graphene after the oxidation process; this leads to a conductance suppression of $\sim 25 \mu \mathrm{S}$ for $\mathrm{V}_{\mathrm{EG}}=0 \mathrm{~V}$ [marked with the red dashed arrows in Fig. 3(f)].

When we applied a positive electrolyte gate voltage of $\mathrm{V}_{\mathrm{EG}}=0.0$ $-0.8 \mathrm{~V}$, a conductance variation of approximately $12-19 \mu \mathrm{S}$ was observed, as shown in Fig. 4(a). The EFM image still shows the electrostatic potential gradient observed in Fig. 4(b). However, upon applying a negative electrolyte gate voltage of $\mathrm{V}_{\mathrm{EG}}=0.0$ to $-1.8 \mathrm{~V}$, a conductance suppression was observed again, but the original conductance was not recovered. After this second oxidation process (3) [Fig. 4(c)], the electrostatic potential and topography images revealed adsorption of undesired residues on the graphene surface, as denoted by the white dashed arrows in Figs. 4(d) and 4(e), respectively. This indicates that the low ratio of oxidized graphene area acts as a seed point for adsorption of residues when $\mathrm{V}_{\mathrm{EG}}=-1.8 \mathrm{~V}$, which is smaller than $\mathrm{V}_{\mathrm{EG}}=-1.95 \mathrm{~V}$ for the first oxidation shown in Fig. 3. Therefore, the oxidation process (1) depicted in Fig. 3 leads to somewhat strong oxidation areas on graphene, which cannot be recovered by the reduction process (2) depicted in Fig. 4(a); moreover, the graphene oxide 
acts as a seed for adsorption of undesired residues, resulting in a conductance suppression (3), as shown in Fig. 4(c). However, these undesired residues adsorbed on graphene oxide could not be confirmed to be only oxides or other residues in impure $\mathrm{H}_{2} \mathrm{SO}_{4}$ electrolyte. Therefore, further investigation is needed to identify these residues.

\section{Conclusions}

In this study, we investigated the redox process occurring on graphene with $\mathrm{H}_{2} \mathrm{SO}_{4}$ as an electrolyte. The graphene surface condition was observed using topography and electrostatic potential images. We observed partial oxidation of the graphene surface even after the reduction process and found that graphene oxide acts as a seed for adsorption of residues on graphene, which leads to conductance suppression. We believe that this result provides a clearer understanding of the redox process on graphene and its continuous conductance degradation.

\section{Acknowledgements}

This work was supported by the research fund of Chungnam National University.

\section{Conflicts of Interest}

The authors declare no conflicts of interest.

\section{ORCID}

Oh Hun Gwon

Seok-Ju Kang

Hye Ryung Byun

Young-Jun Yu

https://orcid.org/0000-0003-2633-7077 https://orcid.org/0000-0003-0337-0165 https://orcid.org/0000-0001-5839-9761 https://orcid.org/0000-0001-9446-1526

\section{References}

[1] J. T. Robinson, F. K. Perkins, E. S. Snow, Z. Wei, and P. E. Sheehan, Nano Lett. 8, 3137 (2008).

[2] L. Guo et al., Carbon 50, 1667 (2012).

[3] C. E. Kehayias, S. MacNaughton, S. Sonkusale, and C. Staii, Nanotechnology 24, 245502 (2013).

[4] M. Cittadini, M. Bersani, F. Perrozzi, L. Ottaviano, W. Wlodarski, and A. Martucci, Carbon 69, 452 (2014).
[5] H. S. Hong, N. H. Phuong, N. T. Huong, N. H. Nam, and N. T. Hue, Appl. Surf. Sci. 492, 449 (2019).

[6] J. Zhang, H. Lu, C. Yan, Z. Yang, G. Zhu, J. Gao, F. Yin, and C. Wang, Sens. Actuators B: Chem. 264, 128 (2018).

[7] W. Zhang, Y. Du, and M. L. Wang, Sens. Bio-Sens. Res. 4, 23 (2015).

[8] X. Kang, J. Wang, H. Wu, I. A. Aksay, J. Liu, and Y. Lin, Biosens. Bioelectron. 25, 901 (2009).

[9] C. Wang et al., Sci. Rep. 6, 21711 (2016).

[10] S. Feng et al., Sci. Adv. 2, e1600322 (2016).

[11] F. Liu, J. Y. Choi, and T. S. Seo, Biosens. Bioelectron. 25, 2361 (2010).

[12] V. K. Gupta, M. L. Yola, M. S. Qureshi, A. O. Solak, N. Atar, and Z. Üstündağ, Sens. Actuators B Chem. 188, 1201 (2013).

[13] Y. Wei, L. Wang, Y. Zhang, and Y. Dong, Sensors 19, 4424 (2019).

[14] I. Khalil, W. A. Yehye, N. M. Julkapli, S. Rahmati, A. A. I. Sina, W. J. Basirun, and M. R. Johan, Biosens. Bioelectron. 131, 214 (2019).

[15] J. Chen, Y. Zhang, M. Zhang, B. Yao, Y. Li, L. Huang, C. Li, and G. Shi, Chem. Sci. 7, 1874 (2016).

[16] A. B. Kaiser, C. Gómez-Navarro, R. S. Sundaram, M. Burghard, and K. Kern, Nano Lett. 9, 1787 (2009).

[17] L. Liu, S. Ryu, M. R. Tomasik, E. Stolyarova, N. Jung, M. S. Hybertsen, M. L. Steigerwald, L. E. Brus, and G. W. Flynn, Nano Lett. 8, 1965 (2008).

[18] X. Wang, L. Zhi, and K. Müllen, Nano Lett. 8, 323 (2008).

[19] Y.-J. Yu, Appl. Sci. Converg. Technol. 27, 35 (2018).

[20] Y.-J. Yu, Appl. Sci. Converg. Technol. 28, 51 (2019).

[21] Y.-J. Yu, Appl. Sci. Converg. Technol. 28, 226 (2019).

[22] Y.-J. Yu, J. Korean Phys. Soc. 74, 132 (2019).

[23] B. G. Sonmez, O. Ertop, and S. Mutlu, Sci. Rep. 7, 12190 (2017)

[24] C. Gómez-Navarro, F. J. Guzmán-Vázquez, J. Gomez-Herrero, J. J. Saenz, and G. M. Sacha, Nanoscale 4, 7231 (2012).

[25] Y.-J. Yu, Y. Zhao, S. Ryu, L. E. Brus, K. S. Kim, and P. Kim, Nano Lett. 9, 3430 (2009).

[26] Y.-J. Yu et al., Nanoscale 11, 4735 (2019).

[27] Y.-J. Yu, M. Y. Han, S. Berciaud, A. B. Georgescu, T. F. Heinz, L. E. Brus, K. S. Kim, and P. Kim, Appl. Phys. Lett. 99, 183105 (2011). 